\title{
A Competition-Based Mechanism Mediates Developmental Refinement of Tectal Neuron Receptive Fields
}

\author{
Wei Dong and Carlos D. Aizenman \\ Department of Neuroscience, Brown University, Providence, Rhode Island 02912
}

Neural activity plays an important role in development and maturation of visual circuits in the brain. Activity can be instructive in refining visual projections by directly mediating formation and elimination of specific synaptic contacts through competition-based mechanisms. Alternatively, activity could be permissive - regulating production of factors that create a favorable environment for circuit refinement. Here we used the Xenopus laevis tadpole visual system to test whether activity is instructive or permissive for shaping development of the retinotectal circuit. In vivo spike output was dampened in a small subgroup of tectal neurons, starting from developmental stages 44 - 46, by overexpressing Shaker-like Xenopus Kv1.1 potassium channels using electroporation. Tadpoles were then reared until stage 49 , a time period when significant refinement of the retinotectal map occurs. Kv1.1-expressing neurons had significantly decreased spike output in response to both current injection and visual stimuli compared to untransfected controls, with spiking occurring during a more limited time interval. We found that Kv1.1-expressing neurons had larger visual receptive fields, decreased receptive field sharpness, and more persistent recurrent excitation than control neurons, all of which are characteristics of immature neurons. Transfected cells, however, had normal spontaneous excitatory synaptic currents and dendritic arbors. These results suggest that spike output of a tectal neuron plays an important instructive role in development of its receptive field properties and refinement of local circuits. However, other activity-dependent processes, such as synaptogenesis and dendritic growth, remain unaffected due to the permissive environment created by otherwise normal network activity.

\section{Introduction}

Sensory projections to the central nervous system are organized as maps representing either sensory space or the organization of the topography of peripheral receptors. This feature is particularly salient in the visual system where retinal projections are organized topographically in their target areas, such that nearby neighboring retinal ganglion cells project to nearby neighboring target neurons. Retinal projections achieve this initial topographic order through molecular guidance cues, and these maps are subsequently refined to mature levels of precision by activitydependent mechanisms (Ruthazer and Cline, 2004; White and Fitzpatrick, 2007; Huberman et al., 2008). However, the role by which activity drives the refinement of these maps is still subject to debate. On one hand, activity could be permissive by regulating the production of factors that create a favorable environment in which circuits can refine. On the other hand, activity could be instructive in that it could directly guide the formation or elimi-

Received May 16, 2012; revised Sept. 10, 2012; accepted Sept. 25, 2012.

Author contributions: W.D. and C.D.A. designed research; W.D. performed research; W.D. and C.D.A. analyzed data; W.D. and C.D.A. wrote the paper.

This work was supported by the National Science Foundation-Integrative Organismal Systems directorate, the National Eye Institute of the NIH, and a Fox Foundation Fellowship for the Visual Sciences (W.D.). We thank Angie Ribera (University of Colorado, Aurora, CO) for the original Kv1.1 construct, Heng Xu and Arto Nurmikko (Brown University, Providence, RI) for providing the visual stimulator, Arseny Khakhalin for useful comments on the manuscript and artwork, and Irina Sears for excellent technical support. The funders had no role in study design, data collection and analysis, decision to publish, or preparation of the manuscript.

Correspondence should be addressed to Carlos D. Aizenman, Brown University, Department of Neuroscience, Box G-LN, Providence, RI 02912. E-mail: Carlos_Aizenman@brown.edu.

DOI:10.1523/JNEUROSCI.2372-12.2012

Copyright $\odot 2012$ the authors $\quad 0270-6474 / 12 / 3216872-08 \$ 15.00 / 0$ nation of specific synaptic contacts through competition-based Hebbian mechanisms. It has proven difficult to design experiments that allow distinction between these two possible roles.

Some clues can be drawn from mammalian studies in the superior colliculus and cortex. For example, pharmacologically disrupting cortical spike output results in abnormally organized cortical columns, suggesting that normal spike output is critical for normal developmental plasticity (Reiter and Stryker, 1988). Similarly, mutant mice with disrupted retinal waves have heavily distorted and expanded visual receptive fields in the superior colliculus (Chandrasekaran et al., 2007; Cang et al., 2008).

One sensory area in which this question can be effectively addressed is the optic tectum of the Xenopus laevis tadpole. The optic tectum is the primary visual area in the frog brain. The projection from the retina to the tectum is retinotopically organized, and its refinement and maintenance are known to depend on visual activity (Debski and Cline, 2002; Ruthazer and Aizenman, 2010). Between developmental stages 45 and 49 , tectal neurons and retinal ganglion cell axons undergo a period of rapid growth and plasticity during which tectal cell dendrites increase in size and complexity and there is increased synaptogenesis and synapse maturation (Wu et al., 1996; Wu et al., 1999; Akerman and Cline, 2006; Pratt and Aizenman, 2007). These processes have been shown to be regulated by visual activity and require activation of a variety of signaling pathways (Wu and Cline, 1998; Cantallops et al., 2000; Sanchez et al., 2006; Bestman and Cline, 2008). During this time period the retinotectal map also refines, such that individual retinal inputs occupy a smaller target area, thus shrinking visual receptive fields (Tao and Poo, 2005; Dong et 
Table 1. Membrane and intrinsic properties of recorded neurons

\begin{tabular}{lccc}
\hline & Control $(n=20)$ & Kv1.1 $(n=20)$ & GFP $(n=8)$ \\
\hline I hold (at $-65 \mathrm{mV})(\mathrm{pA})$ & $18.4 \pm 11.3$ & $33.2 \pm 24.3$ & $14.9 \pm 6.7$ \\
Rinput $(\mathrm{G} \Omega)$ & $2.2 \pm 1.5$ & $1 \pm 0.6$ & $2 \pm 1$ \\
R series $(\mathrm{M} \Omega)$ & $54.8 \pm 19.3$ & $42.1 \pm 15$ & $55.7 \pm 12.7$ \\
Cm (pF) & $14.9 \pm 4.2$ & $28.5 \pm 12.5$ & $16.9 \pm 1.7$ \\
Peak I(Na) $(\mathrm{pA})$ & $524 \pm 68$ & $771 \pm 144$ & $519 \pm 69$ \\
Peak I(K) $(\mathrm{pA})$ & $681 \pm 72$ & $763 \pm 82$ & $612 \pm 69$ \\
\hline
\end{tabular}

A

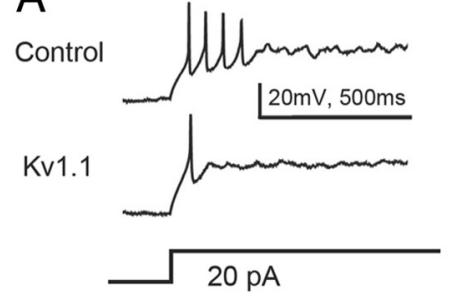

B

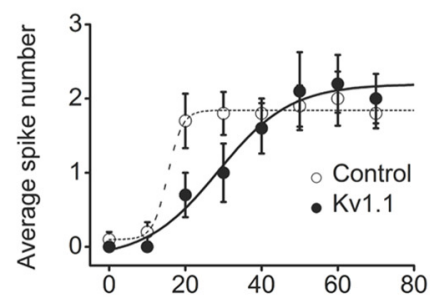

C

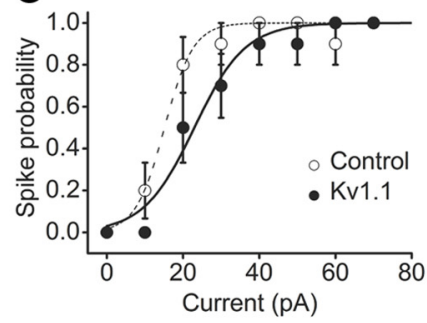

$\mathrm{D}$

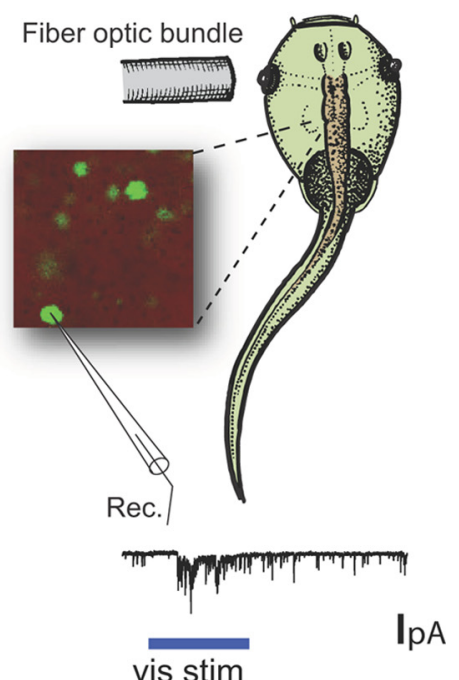

vis stim

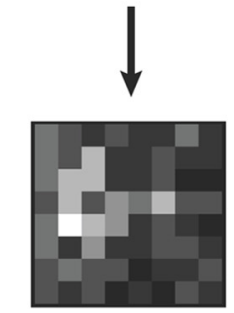

Receptive field map
Figure 1. Transfection of tectal neurons with Xenopus Kv1.1 alters neuronal spike output in response to intracellular current injection. $A$, Sample current-clamp traces showing spike output from a Kv1.1-expressing cell and a control neuron in response to a $20 \mathrm{pA}$ depolarizing pulse. $\boldsymbol{B}$, Input- output curve showing the relationship between injected current and the number of evoked spikes in transfected and control neurons. $N=10$ neurons for each group. Note that control cells spike maximally in response to lower current amplitudes, although maximum output is similar between groups. Curves are statistically significant. C, Same as $\boldsymbol{B}$, but showing relationship between injected current and probability of spiking. Control neurons spike significantly more readily than transfected cells. D, Schematic of recording (Rec.) configuration showing a field of transfected cells in the tectum. In vivo whole-cell or cell-attached recordings were performed from transfected cells to assess spiking at receptive field size. vis stim, Visual stimulation. Error bars are SEM.

al., 2009). Local projections within the tectum also become increasingly organized during this developmental time window, such that recurrent intratectal activity becomes more temporally coherent and less variable (Pratt et al., 2008; Xu et al., 2011). Refinement of both retinotectal afferents and local tectal circuitry has been proposed to be mediated by timing-dependent synaptic plasticity and heavily dependent on correlated patterns of afferent activity (Richards et al., 2010a). However, direct evidence that activity plays an instructive role during this developmental process is still lacking.

Many experimental manipulations that test the role of activity in development of the retinotectal circuit involve either global application of pharmacological agents (Rajan and Cline, 1998; Tao and Poo, 2005; Ruthazer et al., 2006; Xu et al., 2011) or long-term alterations in the sensory environment (Sin et al., 2002; Xu et al., 2011), or they directly interfere with signaling pathways or synaptic transmission (Haas et al., 2006; Sanchez et al., 2006; Bestman and Cline, 2008; Chiu et al., 2008). Thus, these eriments cannot distinguish easily between instructive and issive roles. Other studies focus on showing that patterned activity can acutely alter the temporal response properties and stimulus selectivity of tectal neurons but does not test the instructive role of activity over a longer developmental time scale (Engert et al., 2002; Vislay-Meltzer et al., 2006; Pratt et al., 2008; Richards et al., 2010b). In the present study we use in vivo electroporation to overexpress Shaker-like Xenopus Kv1.1 potassium channels in a small subgroup of tectal neurons in stage 44-46 tadpoles. Overexpression of the channel results in abnormal spike output in these cells in an otherwise intact and normally functioning visual circuit. We test the effects of having altered spike output over a defined developmental time period, comparing transfected cells with untransfected, normally spiking neighbors.

\section{Materials and Methods}

This study was approved by the Brown University Institutional Animal Care and Use Committee (Providence, RI). Brown University has an Animal Welfare Assurance (\#A3284-01) on file with the Office of Laboratory Animal Welfare/NIH. Experiments were performed under protocol \#1007026, approved 8/12/2010. Wild-type Xenopus laevis tadpoles were raised on a $12: 12 \mathrm{~h}$ light:dark cycle at $21^{\circ} \mathrm{C}$ in $10 \%$ Steinberg's solution. Developmental stages of tadpoles were determined according to Nieuwkoop and Faber (1956). Our tadpoles reach stages $44-46$ at $9-2 \mathrm{~d}$ postfertilization and 49 at $18-20 \mathrm{~d}$ postfertilization. At these developmental stages one cannot determine the sex of each animal.

Electroporation and constructs. Whole-brain electroporation (Haas et al., 2002) was used to transfect tectal neurons; tadpoles were anesthetized with $0.02 \%$ MS-222, and plasmid DNA $(3-4 \mu \mathrm{g} / \mu \mathrm{l}$ in water, colored with Fast Green) was pressure injected into the middle ventricle of stage $44-46$ tadpoles using a glass micropipette. Then, two platinum electrodes (1-2 mm) were placed on the skin overlying both sides of the midbrain to electroporate the DNA into the tectal cells. One to two electrical pulses of $50 \mathrm{~V}$ with an exponential decay of $70 \mathrm{~ms}$ were delivered. Immediately after electroporation, the tadpoles were transferred back into a bowl containing normal rearing solution ( $10 \%$ Steinberg's solution). To minimize possible network effects of the transfection, we only selected tadpoles with relatively low transfection rates (5-10 readily identifiable neurons per tectum) to use in electrophysiological recordings. All tectal neuron recordings were done between 3 and $5 \mathrm{~d}$ after electroporation. To overexpress the $\mathrm{K}^{+}$channel, tectal neurons were transfected with a bicistronic EGFP-IRES-Kv1.1 construct (modified from a plasmid kindly provided by Angie Ribera, University of Colorado Health Sciences Center, Denver, CO (Ribera and Nguyen, 1993). For GFP controls, tectal neurons were transfected with pEGFP-C1 vector (Clontech). Drugs and chemicals were obtained from Sigma. 
Electrophysiology. Tadpoles were prepared for in vivo recordings by being first anesthetized with MS-222 $(0.02 \%)$ and then transferred to a recording chamber with HEPESbuffered extracellular medium (containing in mм: $115 \mathrm{NaCl}, 6 \mathrm{KCl}, 3 \mathrm{CaCl} 2,0.5 \mathrm{MgCl} 2,5$ HEPES, $10 \mathrm{~mm}$ glucose; $\mathrm{pH}$ 7.2; osmolarity, $255 \mathrm{mOsm}$ ) in the presence of $0.1 \mathrm{~mm}$ tubocurarine and stabilized with dissecting pins (Aizenman et al., 2003). The tectal lobes were exposed by removing the overlying skin. Cells were visualized using a $\times 60$ water-immersion objective. To reduce any potential variability introduced by the developmental gradient along the rostrocaudal axis, we restricted our recordings to the central third of the tectum (Wu et al., 1996; Khakhalin and Aizenman, 2012). Wholecell, voltage-clamp recordings as well as cellattached recordings were made at room temperature $\left(20-22^{\circ} \mathrm{C}\right)$ using glass micropipettes $(8-12 \mathrm{M} \Omega$ ) filled with intracellular saline (containing in mM: $100 \mathrm{~K}$-gluconate, $8 \mathrm{KCl}, 5$ $\mathrm{NaCl}, 1.5 \mathrm{MgCl}, 20$ HEPES, 10 EGTA, 2 ATP, 0.3 GTP; pH 7.2, osmolarity 255 mOsm). Signals were measured with a Multiclamp 700B amplifier and digitized using a Digidata 1440A A-D board (Molecular Devices). Traces were recorded and analyzed using pClamp 10 software, digitized at $10 \mathrm{kHz}$, and analyzed offline. All chemicals were purchased from Sigma.

Recordings were made from a total of 60 tectal neurons, including 26 transfected with Kv1.1, 26 controls and 8 controls transfected with GFP. Consistent with our prior experience, transfection with control GFP did not have an effect on membrane properties and intrinsic currents, in contrast to transfection with Kv1.1, indicating that the effect was specific to the construct (Table 1). Thus, experiments were performed on Kv1.1-transfected neurons and control cells in Kv1.1-transfected animals to rule out any potential network effects and variability between animals, rather than comparing between GFP and Kv1.1-transfected cells.

Whole-field visual stimulation and receptive field mapping and analysis. To record visually evoked spiking, whole-field flashes of light were presented via a fiber optic cable (Edmund Scientific) attached to a green light-emitting diode (LED; $\lambda_{\max }, 567 \mathrm{~nm}$; Allied Electronics). Light at this wavelength is known to evoke robust visual responses (Aizenman et al., 2003). The fiber optic cable was positioned directly in front of the contralateral eye to stimulate the entire monocular visual field. Visual stimuli were triggered by computer, and stimulus intensity was controlled by varying the voltage across the LED. Extracellular cell-attached responses were recorded in current-clamp mode in response to $1 \mathrm{~s} \mathrm{ON}$ stimuli presented every $10-20 \mathrm{~s}$ and over multiple trials. Visually evoked action potentials were counted over the $1 \mathrm{~s}$ stimulus period. To calculate the spike latency range of visual responses, we pooled all of the spike latencies from a given cell in response to a visual stimulus of a given luminance. We calculated the range of evoked spikes across multiple trials.

To map receptive fields, we used a custom-built blue-green LED array consisting of a $8 \times 8$ grid (Xu et al., 2008). Each LED element had a $25 \mu \mathrm{m}$ diameter with a center-to-center distance of $50 \mu \mathrm{m}$. The array was coupled to a 30,000-pixel, multicore image fiber (FIGH-30-650 S from Fujikura) with a $500 \mu \mathrm{m}^{2}$ image area. This allowed us to project the image of the array to the contralateral eye. The projecting end of the optical fiber was placed near the eye such that the center-to-center spac-
B
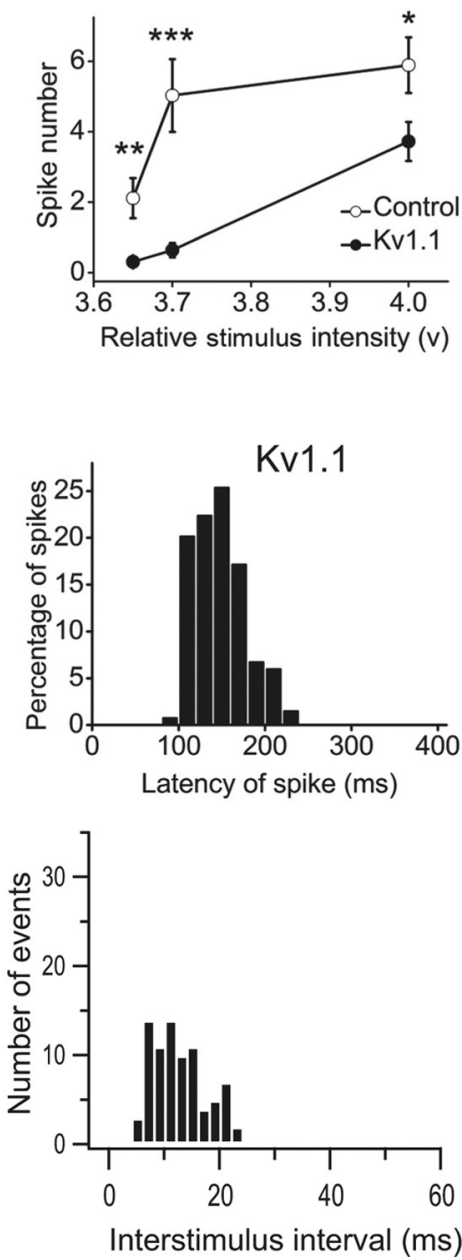

Figure 2. Transfection of tectal neurons with Xenopus Kv1.1 alters neuronal spike output in vivo. $\boldsymbol{A}$, Sample loose cell attached recordings showing visual responses to a whole-field flash comparing a control versus Kv1.1-expressing tectal cell. $\boldsymbol{B}$, Spike output function of stimulus intensity (higher voltage reflects higher luminance) comparing both groups of neurons. $N=6$ for each the broader range of latencies in control neurons, indicating that transfected cells are often not spiking when the rest of the network is active. $\boldsymbol{D}$, Distribution of all interspike intervals for all trials and all cells for control and Kv1.1-expressing groups. Notice the greater diversity of ISIs in the control group. Error bars are SEM; ${ }^{* *} p<0.01,{ }^{* * *} p<0.001$.

ing between the LEDs in the array was about $18^{\circ}$ of arc. Receptive fields were mapped by illuminating individual LEDs for $1 \mathrm{~s}$ during whole-cell recording. Stimuli were presented at $6 \mathrm{~s}$ intervals, and every LED was illuminated at least three times during the duration of the procedure. Whole-cell excitatory synaptic currents resulting from the ON response were averaged for every spatial location, and the integrated synaptic charge was calculated over a period of $1 \mathrm{~s}$.

Results were plotted as image maps, with the brightest square corresponding to the spatial location with the largest response. We defined the peak of the receptive field as the square with the largest response. To measure receptive field size, we counted the number of stimulus locations whose average response was $>50 \%$ of the peak response. To calculate sharpness, we averaged the responses of all the spatial locations immediately adjacent to the peak, including diagonally, and averaged the responses two and three spatial locations away from the peak (Dong et al., 2009). This provided the average rate of decay per distance away from peak. We saw no correlation between receptive field sharpness and location of the receptive field peak, suggesting that this is a useful metric to assess the spatial decay of tectal receptive fields regardless of location (Dong et al., 2009). Values were normalized to the peak value and then fit with an exponential decay using the least-squares method. An $F$ test was 
A

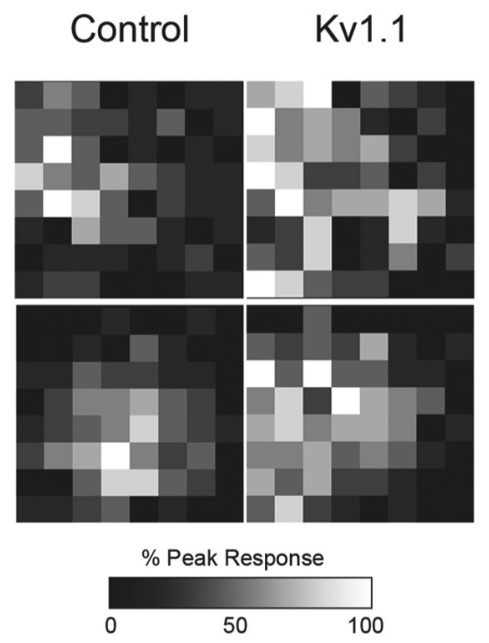

B

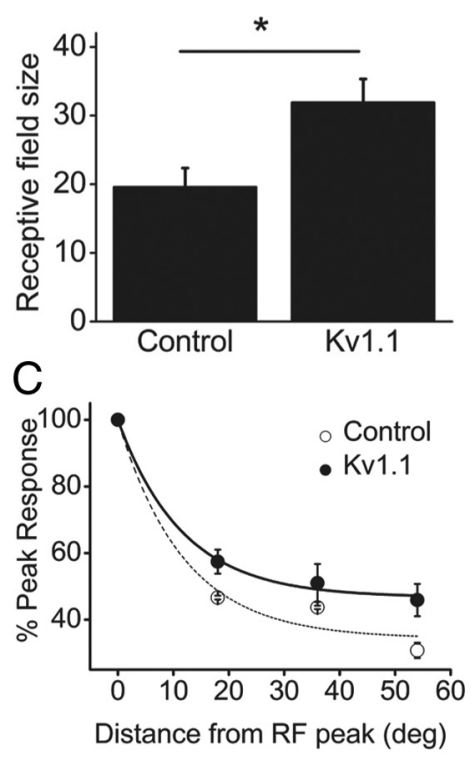

Figure 3. Kv1.1 overexpression results in expanded visual receptive fields. $A$, Sample receptive field maps of two control and two Kv1.1-expressing tectal neurons. Each square represents the response to a single LED at the indicated location; brightness scale indicates size of the response. The center-to-center distance of each LED corresponds to $18^{\circ}$ of arc. $\boldsymbol{B}$, Average receptive field (RF) size for control ( $n=6)$ and Kv1.1-expressing $(n=7)$ neurons. C, Average "sharpness" of receptive fields indicating a significantly sharper drop-off in response size in control versus transfected neurons, $p=0.0032$. Error bars are SEM; ${ }^{*} p=0.0187$.

A

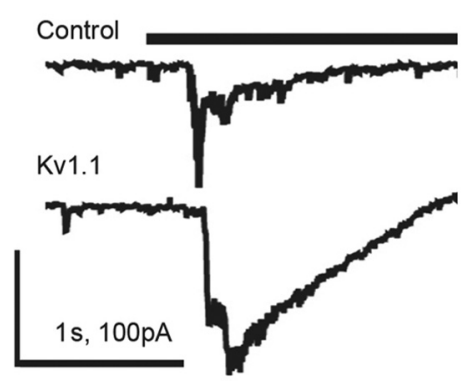

B

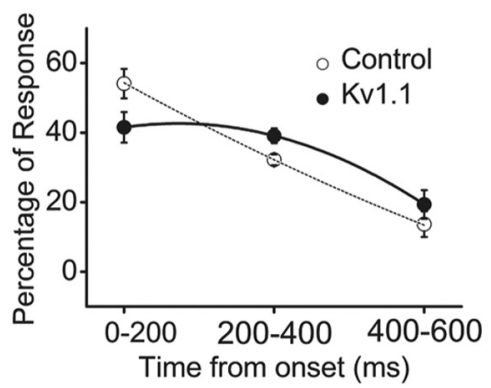

Figure 4. Kv1.1 overexpression results in lack of temporal refinement of local tectal circuits. $A$, Sample voltage-clamp recording of excitatory currents evoked by visual stimuli reflecting polysynaptic recurrent network activity. Note the shorter duration of the recurrent activity in control neurons. $\boldsymbol{B}$, Temporal distribution of the total evoked charge (area under the curve) of visual responses in control $(n=6)$ and Kv1.1-expressing $(n=7)$ neurons. Note the significantly different distribution of the response shape in transfected neurons, indicating a longer lasting recurrent response, $p=0.035$. Error bars are SEM.

used to determine statistical significance by testing whether the two data sets were best fit by a single curve or by two different curves (Dong et al., 2009). To calculate temporal decay, we used peak responses and integrated the charge over $200 \mathrm{~ms}$ bins for the first $600 \mathrm{~ms}$. The decay was best fit to a curve, and the curves were compared using an $F$ test. Statistics and curve fitting were done using Prism 5 from GraphPad Software.

Morphology. A total of 13 tectal cells were successfully reconstructed. Individual Kv1.1-expressing or control tectal cells were impaled with a sharp micropipette (impedance $\approx 150 \mathrm{M} \Omega$ ) containing $1 \%$ Lucifer Yellow and $4 \%$ neurobiotin (Vector Laboratories) and injected continuously with $+1 \mathrm{nA}$ for $5 \mathrm{~min}$. The whole brain preparations were then fixed in $4 \%$ paraformaldehyde for $1 \mathrm{~h}$ and washed several times with $0.1 \mathrm{M}$ PBS/0.5\% Triton X-100. Neurobiotin-injected tectal cells were visualized by incubating the brain in $5 \%$ streptavidin-FITC in $0.1 \mathrm{M} \mathrm{PBS} / 0.5 \%$ Triton X-100 overnight at $4^{\circ} \mathrm{C}$ and then washed 3 times with $0.1 \mathrm{M} \mathrm{PB}$.

A Zeiss LSM-510 Meta confocal microscope was used to image whole brain preparations containing labeled neurons. Optical sections were acquired from the confocal microscope at 1 $\mu \mathrm{m}$ intervals using a $63 \times$ objective. The confocal images were then used to reconstruct the 3D morphology of tectal cells using Neuromantic software (http://www.reading.ac.uk/ neuromantic/). Three-dimensional reconstructions of dendritic arbors were created by Neuromantic software and were used to calculate the total dendritic branch length, branch numbers, and tip numbers. L-Measure (http://cng.gmu.edu: $8080 / \mathrm{Lm} /$ ) was used for three-dimensional Sholl analysis. The number of dendritic branches that intersect each sphere was summed in each 10 $\mu \mathrm{m}$ bin for plotting and statistical analysis. All morphometric data except Sholl analysis were tested for significance by using unpaired Student's $t$ tests.

\section{Results}

To examine how the neuronal activity affects the refinement of retinotectal projection, we overexpressed Shaker-like Kv1.1 potassium channels in tectal neurons of stage 44-46 tadpoles by in vivo electroporation (Haas et al., 2002). This technique allows us to selectively impair excitability in a small subset of neurons without affecting the overall levels of activity in the tectal circuit. The rationale underlying the choice of this channel was to find a manipulation that would decrease total spike output but be sufficiently subtle to prevent strong homeostatic compensation of intrinsic currents that would ultimately restore spiking to normal levels, as has been observed following transfection of inwardly rectifying $\mathrm{K}^{+}$channels in this preparation (Pratt and Aizenman, 2007). The intrinsic excitability of transfected neurons was tested by performing wholecell recordings during stage $48 / 9$, when substantial refinement of the retinotectal map has occurred (Dong et al., 2009). Excitability was assayed by injecting square current pulses of varying amplitude and measuring resulting spikes in currentclamp configuration while holding the cell at $-65 \mathrm{mV}$ with negative current injection. We found that both Kv1.1expressing and control neurons showed similar levels of spiking in response to large current injection (at 70 pA current injection: Kv1.1, $2.0 \pm 0.3$ spikes; Control, $1.8 \pm$ 0.2 spikes; $n=10$ ). However, during small current injections Kv1.1-expressing neurons showed significantly reduced spiking compared with control neurons (at 20 pA current injection, Fig. $1 A ; \mathrm{Kv} 1.1,0.7 \pm 0.3$ spikes; control, $1.7 \pm 0.4$ spikes; $p=0.04$, unpaired $t$ test, $n=10$ ). This reduction in spike output is consistent with the expected effect of overexpressing Kv1.1. When we compared the input-output curves (Fig. $1 B, C$ ) by plotting either the number of spikes or the probability of spiking versus amplitude of injected current, we found the curves to be significantly different between groups, with the Kv1.1 cells showing decreased excitability (number of spikes, $p=0.008$; spike probability, $p=$ $0.001, F$ test). This indicates that Kv1.1 expression impairs the 
intrinsic excitability of tectal cells without affecting the neuron's maximal spike output.

We next tested whether spike output in response to a whole-field visual stimulus was affected by Kv1.1 expression. In vivo cell-attached recordings (see Fig. $1 D$ for experimental schematic) indicate that Kv1.1-expressing neurons show overall fewer spikes in response to a range of visual stimuli of varying intensity, with significantly fewer spikes evoked at each stimulus intensity (Fig. $2 A, B$; e.g., for a $3.7 \mathrm{~V}$ stimulus, control: $5.03 \pm 1.03$ spikes, $n=6$; Kv1.1: $0.64 \pm 0.20$ spikes, $n=6 ; p=0.001$, unpaired $t$ test). The overall duration of the spiking response was also reduced in transfected cells. The aggregate distribution of evoked spike latencies, that is, the latency to each spike from the onset of the visual stimulus, shows a significant difference between the two distributions in all recordings (Fig. $2 C ; p<0.0001$, Kolmogorov-Smirnov test), indicating that Kv1.1-expressing cells fire at a more limited range of latencies than control neurons in response to a visual stimulus and therefore will often not be firing while control neurons remain active. Likewise, a comparison of the distribution of interspike intervals following a visual stimulus shows significant differences between the control and the Kv1.- transfected group (Fig. $2 D ; p=$ 0.026 Kolmogorov-Smirnov test). Thus, Kv1.1 transfection alters visual responses by decreasing the number of evoked spikes, limiting the duration of the visual response, and altering the timing between evoked spikes relative to controls.

During early development of the optic tectum, there is significant refinement of tectal neuron receptive field size. Gross manipulations of neural activity suggest that this refinement is dependent on activity. Thus, we tested whether Kv1.1-transfected neurons, in which normal spiking is impaired, had larger visual receptive fields relative to untransfected neurons, indicating abnormal developmental refinement. To map receptive fields, we performed whole-cell, patch-clamp recordings on tectal neurons in vivo while presenting patterned visual stimuli to the contralateral eye. Excitatory synaptic currents were isolated by holding the membrane potential at $-45 \mathrm{mV}$, the reversal potential for GABARmediated currents. The stimuli were generated with a $8 \times 8$ microLED array and delivered to the eye by a fiber optic bundle (see Materials and Methods; Xu et al., 2008). We compared the receptive field map for both Kv1.1-expressing and control tectal neurons (Fig. $3 A$ ). We calculated the size of the receptive field as percentage of stimulus locations that had response amplitudes larger than half of the peak response. The receptive field size of Kv1.1-expressing neurons was significantly larger than that in control neurons (Fig. 3A; Kv1.1, 31.9 $\pm 3.4 \%, n=7$; control, $19.6 \pm 2.7 \%, n=6 ; p=0.019$, unpaired $t$ test). We also analyzed the "sharpness" of the receptive field and the temporal profile of the visual responses (Dong et al., 2009). The sharpness was calculated as the average decay of response size (measured as total integrated synaptic charge) as a function of
Control
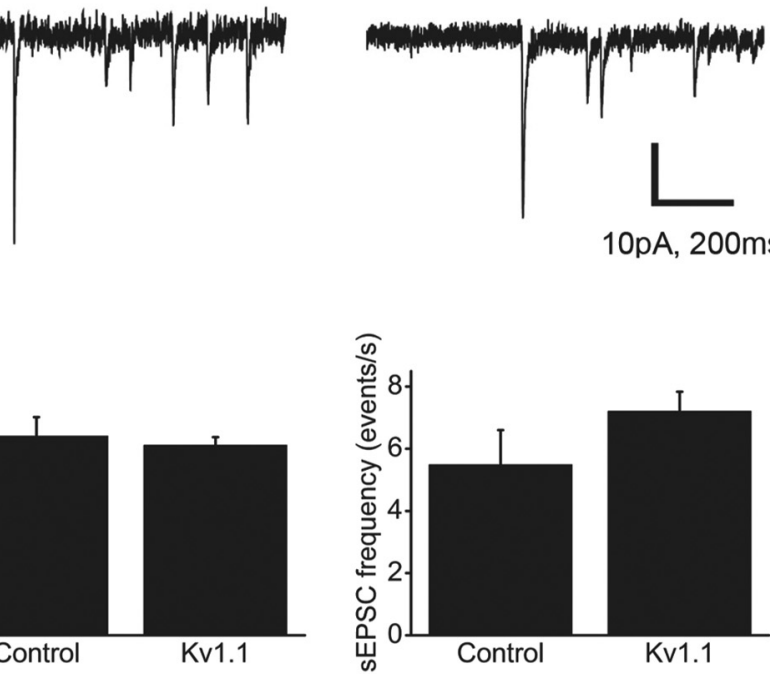

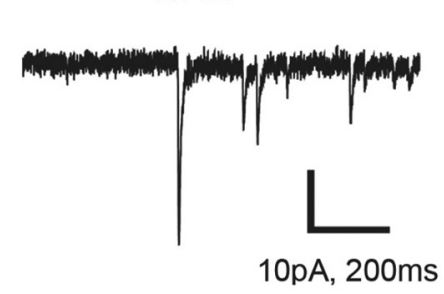

10pA, 200ms

Kv1.1
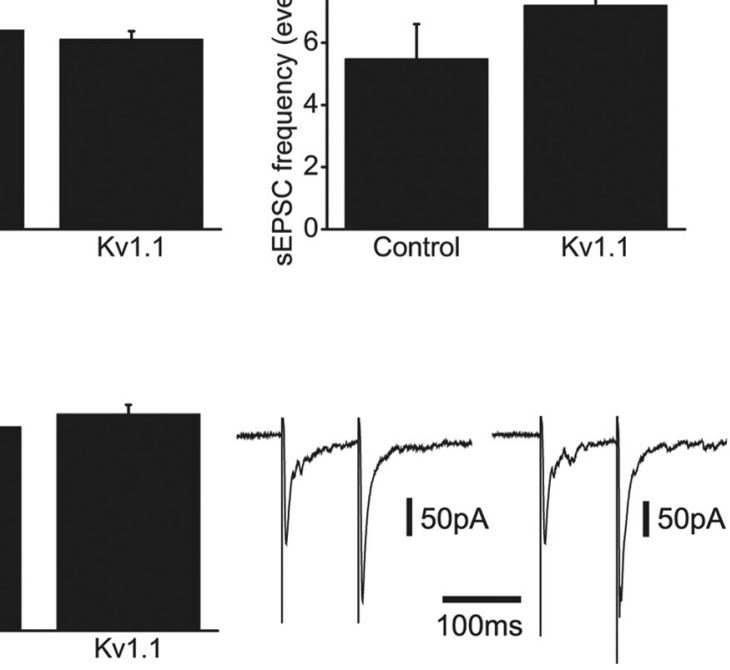

Figure 5. Kv1.1 overexpression does not affect normal development of spontaneous excitatory synaptic transmission. $A$, Sample spontaneous EPSCs from control and Kv1.- transfected neurons. $\boldsymbol{B}$, Average amplitude and frequency of spontaneous EPSCs is not different between groups. $C$, Amount of paired pulse stimulation is also not different between groups. Traces show averaged responses from a representative control (left) and Kv1.1-expressing (right) neuron. Error bars are SEM.

distance from the receptive field peak. Kv1.1-expressing neurons had significantly fewer sharp receptive fields compared with controls; at one LED element away from the peak $\left(\approx 18^{\circ}\right.$ of arc), the average response size of Kv1.1-transfected neurons had decreased to $57.4 \pm 3.6 \%$ of peak $(n=7)$, whereas in control neurons the response decreased to $46.6 \pm 0.6 \%$ of peak $(n=6)$. Moreover, when we compared the overall decay of the visual response as a function of distance from the receptive field peak, the curve fits between transfected and control neurons were significantly different (Fig. 3C; $p=$ $0.003, F$ test), with Kv1.1-expressing neurons showing a longer decay, indicating an expanded receptive field size. Together, these results show that cells with impaired spiking due to Kv1.1 expression have a lack of developmental refinement of receptive fields.

The temporal response properties of visual responses in the tectum are also known to change over development as a result of the refinement of local tectal circuits (Pratt et al., 2008). Refinement of local circuits can also contribute to refinement in tectal cell receptive field size (Zhang et al., 2011). This refinement can be detected as a change in recurrent intratectal activity, such that this activity becomes progressively faster and more consistent over development. We found that the temporal profile of visual responses was also changed by Kv1.1 overexpression (Fig. $4 A$ ). To calculate the temporal profile of visual responses, we integrated the synaptic charge during three $200 \mathrm{~ms}$ bins in a $600 \mathrm{~ms}$ time window following the onset of the response and expressed this measure as a percentage of the total synaptic charge over this time period. Kv1.1-expressing neurons showed relatively flat temporal 

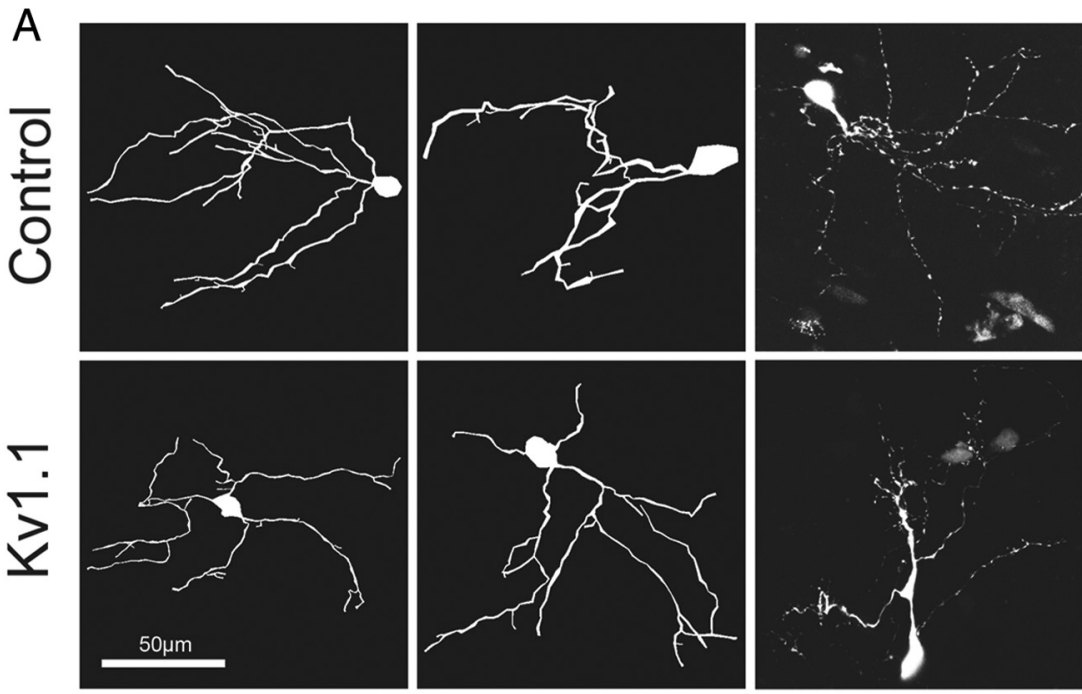

B
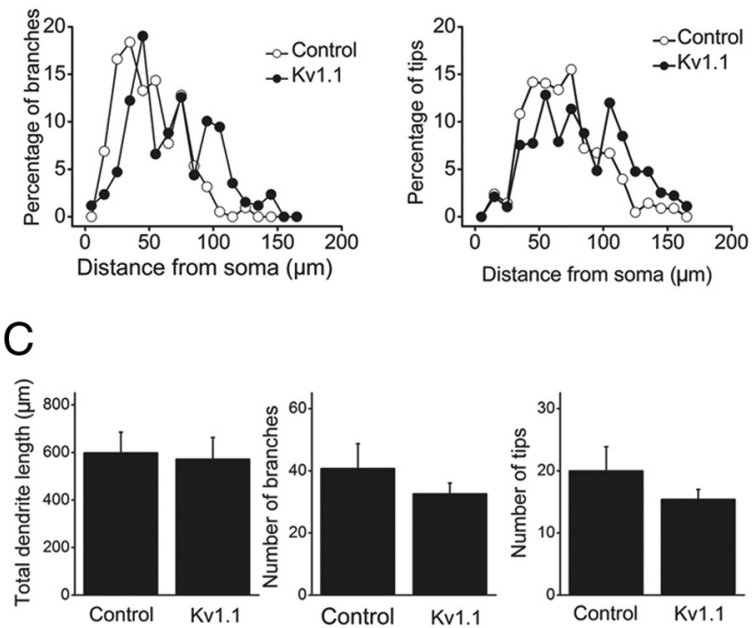

Figure 6. Kv1.1 overexpression does not affect development of tectal cell dendritic morphology. A, Sample reconstructions of tectal cell dendrites from control and Kv1.1 transfected neurons. Photomicrographs on the right show maximum z-series projections of example labeled neurons. Cells in the background are cell bodies of transfected but nonfilled neurons. Example cell in the control image has some distal dendrites cut off to fit in the figure, but reconstruction and analysis were performed on entire arbors. $B$, Scholl-like analysis from grouped data ( $n=6$ for control, $n=5$ for Kv1.1) measuring percentage of branches and branch tips as a function of distance from the soma. No significant differences were observed between the distributions from both groups. $C$, Summary data measuring total dendritic branch length, branch number, and number of tips between groups. No differences were seen between control and Kv1.1-transfected groups, indicating that dendritic development appears normal. Error bars are SEM.

profile compared with control neurons during the first two time bins, indicating a shift in the temporal distribution of recurrent excitatory synaptic activity and consistent with what is observed in more immature neurons. The polysynaptic component of visual responses in Kv1.1-expressing neurons decayed from $41.5 \pm$ $4.4 \%$ of the total response to $39.1 \pm 2.1 \%(n=7)$ in the first two bins, whereas those of control neurons decayed from $54.3 \pm 4.2$ to $32.2 \pm 1.6 \%(n=6)$. The overall shape of the temporal distribution was significantly different between both groups, as shown by the significant differences in the fitted curves (Fig. $4 B ; p=$ $0.035, F$ test). This finding suggests that Kv1.1 expression also results in lack of refinement of local tectal circuits in transfected neurons, leading to prolonged and more persistent polysynaptic activity.

Development of synaptic transmission and dendritic growth in tectal neurons is also known to be regulated by neural activity
(Sin et al., 2002; Aizenman and Cline, 2007). Thus, we tested whether these parameters were altered in Kv1.1-expressing neurons. To assess synaptic activity, we measured spontaneous EPSCs (sEPSCs; Fig. 5A) and calculated both the frequency and amplitude as indicators of total excitatory synaptic drive. The frequency is proportional to the number of synapses received by the cell, while the amplitude reflects synaptic strength. We did not find any significant differences in either parameter (Fig. 5B; Kv1.1, $7.2 \pm 0.6$ events/s, $-7.2 \pm 0.3 \mathrm{pA}, n=7$; Control, $5.5 \pm 1.1$ events/s, $-7.5 \pm 0.7 \mathrm{pA}, n=6$ ). We further tested whether Kv1.1 transfection affected synaptic release to the transfected cell by performing paired-pulse experiments. Paired synaptic stimuli use direct electrical stimulation of the optic nerve. A change in presynaptic release probability is expected to result in a change in the ratio of the first to the second evoked EPSC. We found no difference between Kv1.1-expressing cells and controls (Fig. 5C; EPSC2/EPSC1; Kv1.1, $1.65 \pm 0.07, n=8$; Control, $1.55 \pm 0.1$, $n=7$ ).

To test whether Kv1.1 expression affected the morphological development of the tectal neuron dendritic arbor, we revealed the dendritic morphology of both Kv1.1-expressed neurons and control neurons by intracellular injection of Lucifer Yellow and Neurobiotin into the soma. The 3D structure of dendritic tree was reconstructed and analyzed (Fig. 6A). First, we examined the morphology of the dendritic tree using a 3D Sholl-like analysis, calculating the percentage of branches and tips as a function of the distance from soma. We found no significant differences in the distribution of branches and branch tips between groups (Fig. 6B). We also examined the total dendritic length, number of branches, and number of tips of both Kv1.1 and control neurons. We did not find any changes in these morphological properties after Kv1.1 expression (Fig. 6C; total dendritic length, $572.2 \pm 91.0$ vs $598.4 \pm 86.7 \mu \mathrm{m}$; number of branches, $32.6 \pm 3.4$ vs $40.7 \pm 8.0$; number of tips, $15.4 \pm 1.6$ vs $20.0 \pm 3.8$. for $\operatorname{Kv1.1}(n=5)$ and control $(n=7)$, respectively). Taken together, these data indicate that Kv1.1 expression does not affect dendritic growth and synaptogenesis.

\section{Discussion}

The role of activity in development of the retinotectal circuit has been studied extensively at the level of the entire network, using global manipulations of neural activity (Ruthazer and Aizenman, 2010). However, these manipulations make it impossible to distinguish between the permissive and instructive roles of activity. We used genetic manipulations to alter the activity of a small subset of tectal neurons by overexpressing Kv1.1 during a critical 
developmental window. Our findings show that tectal neurons that overexpress Kv1.1 during development have enlarged visual receptive fields and prolonged, persistent recurrent excitation compared to control neurons. Transfected cells, however, did not show differences in spontaneous excitatory transmission or in dendritic morphology. These observations are consistent with an interpretation in which activity-dependent pruning of retinotectal afferents and local intratectal projections is abnormal, while other activity-dependent aspects of tectal cell development, such as synaptogenesis and dendritic development, appear to be unaffected.

These findings may serve to highlight different roles of neural activity in early circuit development. Activity is thought to play an instructive role in the formation of sensory maps in a diversity of species and brain regions where the spatiotemporal patterning of the sensory stimuli is important for guiding the pruning and stabilization of neural connections (Knudsen, 2002; White and Fitzpatrick, 2007; Huberman et al., 2008; Ruthazer and Aizenman, 2010). Thus, consistent with this competition-based model, cells transfected with Kv1.1, which fire fewer spikes in response to visual stimuli and over a narrower time interval than neighboring untransfected cells, will therefore fail to refine at the same rate as the overall circuit. Since refinement and pruning of local intratectal connections are also highly dependent on the temporal aspects of activity (Pratt et al., 2008), recurrent excitation also fails to develop normally in transfected neurons. Because the efficiency of transfection is very low under our experimental conditions (about 5-10 neurons per tectum), this is not expected to significantly alter network dynamics, limiting the effects on refinement to Kv1.1-expressing cells and not affecting development of the retinotectal circuit as a whole.

Other activity-dependent aspects of tectal cell development appear to develop normally in Kv1.1-expressing cells. Both dendritic morphology and spontaneous excitatory transmission appear normal. While these are not exhaustive tests of synaptic and dendritic development, they do indicate that on a general level transfected cells are not different from controls. Development of the dendritic arbor and synaptogenesis likely reflects the permissive role of neural activity in development. In Xenopus tadpoles, both synaptic maturation and dendritic development are regulated by a variety of activity-dependent processes like expression of genes such as cpg15 and homer 1A (Cantallops et al., 2000; Van Keuren-Jensen and Cline, 2008); signaling pathways such as those involving CaMKII, calcineurin/NFAT, and Rho GTPases (Wu and Cline, 1998; Li et al., 2002; Schwartz et al., 2009); and CPEB-mediated local protein translation (Bestman and Cline, 2008). Since Kv1.1 expression does not completely prevent cells from spiking, and spiking appears normal in response to strong stimuli, it is possible that this spiking is sufficient to maintain activation of these pathways. Synaptic input to transfected cells also remains unaltered, and this could contribute to activating these pathways independently of neuronal spiking. This might also explain the finding of Haas et al. (2006), in which directly interfering with AMPA receptor trafficking results in cells with smaller dendritic arbors and immature synapses. Furthermore, synaptic and dendritic development is also influenced by activitydependent release of diffusible factors, such as BDNF and nitric oxide (Rentería and Constantine-Paton, 1999; Cogen and Cohen-Cory, 2000; Tu and Debski, 2001; Sanchez et al., 2006; Schwartz et al., 2011). Since only a small fraction of cells is transfected, these factors would still be released by the surrounding untransfected cells and thus not be affected by Kv1.1 expression.

Similar to our prior finding that transfecting tectal neurons with inwardly rectifying $\mathrm{K}$ channels does not result in a homeostatic compensation of excitatory transmission (Pratt and Aizenman, 2007), and in contrast to studies in the hippocampus (Burrone et al., 2002), we found that transfecting cells with Kv1.1 does not cause compensatory changes in sEPSC size, frequency, or paired pulse facilitation. However, we did see some compensation in voltage-gated $\mathrm{Na}^{2+}$ currents. This suggests that our manipulation is sufficiently subtle to not trigger synaptic scaling, which can be induced by directly interfering with synaptic transmission (Deeg and Aizenman, 2011), but can trigger homeostatic plasticity of intrinsic currents. This compensation, however, was not sufficient to restore evoked spiking to its normal levels.

Our findings complement those from zebrafish embryos in which activity of individual retinal ganglion cells was genetically reduced, and their axonal arbors in the tectum were found to be smaller than those of their untransfected neighbors (Hua et al., 2005). This suggests that blocking activity in select cells, either presynaptically or postsynaptically, results in lack of input refinement in the tectum. However, findings in the tectum stand in contrast to results from rat somatosensory cortex, where dampening spiking in subsets of layer $2 / 3$ pyramidal neurons resulted in reduced synaptic drive but normal development of receptive fields (Komai et al., 2006). This suggests that competition-based refinement may not be a universal feature of circuit development.

Taken together, our findings support a model in which early visual activity instructs the refinement of retinotectal and intratectal connections, causing the shrinking of tectal neuron receptive fields. Interfering with the normal spike output of the neuron interferes with this competition-based mechanism, causing abnormal development of receptive fields and recurrent projections. However, dendritic growth and synaptogenesis either do not require spike output and are mediated by synaptic signaling, or the residual spiking and normal network activity are sufficient to maintain these permissive activity-dependent processes. These experiments highlight both the instructive and permissive roles of activity in visual system development.

\section{References}

Aizenman CD, Cline HT (2007) Enhanced visual activity in vivo forms nascent synapses in the developing retinotectal projection. J Neurophysiol 97:2949-2957. CrossRef Medline

Aizenman CD, Akerman CJ, Jensen KR, Cline HT (2003) Visually driven regulation of intrinsic neuronal excitability improves stimulus detection in vivo. Neuron 39:831-842. CrossRef Medline

Akerman CJ, Cline HT (2006) Depolarizing GABAergic conductances regulate the balance of excitation to inhibition in the developing retinotectal circuit in vivo. J Neurosci 26:5117-5130. CrossRef Medline

Bestman JE, Cline HT (2008) The RNA binding protein CPEB regulates dendrite morphogenesis and neuronal circuit assembly in vivo. Proc Natl Acad Sci U S A 105:20494-20499. CrossRef Medline

Burrone J, O’Byrne M, Murthy VN (2002) Multiple forms of synaptic plasticity triggered by selective suppression of activity in individual neurons. Nature 420:414-418. CrossRef Medline

Cang J, Wang L, Stryker MP, Feldheim DA (2008) Roles of ephrin-as and structured activity in the development of functional maps in the superior colliculus. J Neurosci 28:11015-11023. CrossRef Medline

Cantallops I, Haas K, Cline HT (2000) Postsynaptic CPG15 promotes synaptic maturation and presynaptic axon arbor elaboration in vivo. Nat Neurosci 3:1004-1011. CrossRef Medline

Chandrasekaran AR, Shah RD, Crair MC (2007) Developmental homeostasis of mouse retinocollicular synapses. J Neurosci 27:1746-1755. CrossRef Medline

Chiu SL, Chen CM, Cline HT (2008) Insulin receptor signaling regulates synapse number, dendritic plasticity, and circuit function in vivo. Neuron 58:708-719. CrossRef Medline

Cogen J, Cohen-Cory S (2000) Nitric oxide modulates retinal ganglion cell 
axon arbor remodeling in vivo. J Neurobiol 45:120-133. CrossRef Medline

Debski EA, Cline HT (2002) Activity-dependent mapping in the retinotectal projection. Curr Opin Neurobiol 12:93-99. CrossRef Medline

Deeg KE, Aizenman CD (2011) Sensory modality-specific homeostatic plasticity in the developing optic tectum. Nat Neurosci 14:548-550. CrossRef Medline

Dong W, Lee RH, Xu H, Yang S, Pratt KG, Cao V, Song YK, Nurmikko A, Aizenman CD (2009) Visual avoidance in Xenopus tadpoles is correlated with the maturation of visual responses in the optic tectum. J Neurophysiol 101:803-815. CrossRef Medline

Engert F, Tao HW, Zhang LI, Poo MM (2002) Moving visual stimuli rapidly induce direction sensitivity of developing tectal neurons. Nature 419: 470-475. CrossRef Medline

Haas K, Jensen K, Sin WC, Foa L, Cline HT (2002) Targeted electroporation in Xenopus tadpoles in vivo-from single cells to the entire brain. Differentiation 70:148-154. CrossRef Medline

Haas K, Li J, Cline HT (2006) AMPA receptors regulate experiencedependent dendritic arbor growth in vivo. Proc Natl Acad Sci U S A 103: 12127-12131. CrossRef Medline

Hua JY, Smear MC, Baier H, Smith SJ (2005) Regulation of axon growth in vivo by activity-based competition. Nature 434:1022-1026. CrossRef Medline

Huberman AD, Feller MB, Chapman B (2008) Mechanisms underlying development of visual maps and receptive fields. Annu Rev Neurosci 31: 479-509. CrossRef Medline

Khakhalin AS, Aizenman CD (2012) GABAergic transmission and chloride equilibrium potential are not modulated by pyruvate in the developing optic tectum of Xenopus laevis tadpoles. PLoS One 7:e34446. CrossRef Medline

Knudsen EI (2002) Instructed learning in the auditory localization pathway of the barn owl. Nature 417:322-328. CrossRef Medline

Komai S, Licznerski P, Cetin A, Waters J, Denk W, Brecht M, Osten P (2006) Postsynaptic excitability is necessary for strengthening of cortical sensory responses during experience-dependent development. Nat Neurosci 9:1125-1133. CrossRef Medline

Li Z, Aizenman CD, Cline HT (2002) Regulation of rho GTPases by crosstalk and neuronal activity in vivo. Neuron 33:741-750. CrossRef Medline

Nieuwkoop PD, Faber J (1956) Normal table of Xenopus laevis (Daudin). New York: Garland.

Pratt KG, Aizenman CD (2007) Homeostatic regulation of intrinsic excitability and synaptic transmission in a developing visual circuit. J Neurosci 27:8268-8277. CrossRef Medline

Pratt KG, Dong W, Aizenman CD (2008) Development and spike timingdependent plasticity of recurrent excitation in the Xenopus optic tectum. Nat Neurosci 1:467-475. CrossRef Medline

Rajan I, Cline HT (1998) Glutamate receptor activity is required for normal development of tectal cell dendrites in vivo. J Neurosci 18:7836-7846. Medline

Reiter HO, Stryker MP (1988) Neural plasticity without postsynaptic action potentials: less-active inputs become dominant when kitten visual cortical cells are pharmacologically inhibited. Proc Natl Acad Sci U S A 85:36233627. CrossRef Medline

Rentería RC, Constantine-Paton M (1999) Nitric oxide in the retinotectal system: a signal but not a retrograde messenger during map refinement and segregation. J Neurosci 19:7066-7076. Medline

Ribera AB, Nguyen DA (1993) Primary sensory neurons express a Shakerlike potassium channel gene. J Neurosci 13:4988-4996. Medline
Richards BA, Aizenman CD, Akerman CJ (2010a) In vivo spike-timingdependent plasticity in the optic tectum of Xenopus laevis. Front Synaptic Neurosci 2:7. CrossRef Medline

Richards BA, Voss OP, Akerman CJ (2010b) GABAergic circuits control stimulus-instructed receptive field development in the optic tectum. Nat Neurosci 13:1098-1106. CrossRef Medline

Ruthazer ES, Aizenman CD (2010) Learning to see: patterned visual activity and the development of visual function. Trends Neurosci 33:183-192. CrossRef Medline

Ruthazer ES, Cline HT (2004) Insights into activity-dependent map formation from the retinotectal system: a middle-of-the-brain perspective. J Neurobiol 59:134-146. CrossRef Medline

Ruthazer ES, Li J, Cline HT (2006) Stabilization of axon branch dynamics by synaptic maturation. J Neurosci 26:3594-3603. CrossRef Medline

Sanchez AL, Matthews BJ, Meynard MM, Hu B, Javed S, Cohen Cory S (2006) BDNF increases synapse density in dendrites of developing tectal neurons in vivo. Development 133:2477-2486. CrossRef Medline

Schwartz N, Schohl A, Ruthazer ES (2009) Neural activity regulates synaptic properties and dendritic structure in vivo through calcineurin/NFAT signaling. Neuron 62:655-669. CrossRef Medline

Schwartz N, Schohl A, Ruthazer ES (2011) Activity-dependent transcription of BDNF enhances visual acuity during development. Neuron 70 : 455-467. CrossRef Medline

Sin WC, Haas K, Ruthazer ES, Cline HT (2002) Dendrite growth increased by visual activity requires NMDA receptor and Rho GTPases. Nature 419:475-480. CrossRef Medline

Tao HW, Poo MM (2005) Activity-dependent matching of excitatory and inhibitory inputs during refinement of visual receptive fields. Neuron 45:829-836. CrossRef Medline

Tu S, Debski EA (2001) Neurotrophins, but not depolarization, regulate substance P expression in the developing optic tectum. J Neurobiol 48: 131-149. CrossRef Medline

Van Keuren-Jensen KR, Cline HT (2008) Homer proteins shape Xenopus optic tectal cell dendritic arbor development in vivo. Dev Neurobiol 68: 1315-1324. CrossRef Medline

Vislay-Meltzer RL, Kampff AR, Engert F (2006) Spatiotemporal specificity of neuronal activity directs the modification of receptive fields in the developing retinotectal system. Neuron 50:101-114. CrossRef Medline

White LE, Fitzpatrick D (2007) Vision and cortical map development. Neuron 56:327-338. CrossRef Medline

Wu GY, Cline HT (1998) Stabilization of dendritic arbor structure in vivo by CaMKII. Science 279:222-226. CrossRef Medline

Wu GY, Zou DJ, Rajan I, Cline H (1999) Dendritic dynamics in vivo change during neuronal maturation. J Neurosci 19:4472-4483. Medline

Wu G, Malinow R, Cline HT (1996) Maturation of a central glutamatergic synapse. Science 274:972-976. CrossRef Medline

Xu H, Davitt KM, Dong W, Song YK, Patterson WR, Aizenman CD, Nurmikko AV (2008) Combining multicore imaging fiber with matrix addressable blue/green LED arrays for spatiotemporal photonic excitation at cellular level. IEEE J Sel Top Quant Electron 14:167-170. CrossRef

$\mathrm{Xu}$ H, Khakhalin AS, Nurmikko AV, Aizenman CD (2011) Visual experience-dependent maturation of correlated neuronal activity patterns in a developing visual system. J Neurosci 31:8025-8036. CrossRef Medline

Zhang M, Liu Y, Wang SZ, Zhong W, Liu BH, Tao HW (2011) Functional elimination of excitatory feedforward inputs underlies developmental refinement of visual receptive fields in zebrafish. J Neurosci 31:5460-5469. CrossRef Medline 